\title{
Small-molecule disruptors of glucokinase inhibition
}

Genome-wide association studies have indicated that polymorphisms in the gene $G C K R$, which encodes glucokinase regulatory protein (GCKR), reduce the binding affinity of this inhibitor for glucokinase. In a recent publication in Nature, Amgen researchers show that pharmacological intervention can do the same, at least in rodents.

The GCKR-mediated inhibitory control of glucokinase is well-documented. Individuals that express GCKR variants with lower binding affinity for glucokinase, exhibit reduced blood glucose levels and a lower incidence of type 2 diabetes mellitus (T2DM) than the general population.

The discovery of glucokinase activators first put glucokinase in the limelight as a compelling target for the treatment of T2DM. However, one of the biggest problems of agents that target glucokinase directly has been the incidence and severity of hypoglycaemia.

"We decided to target liver glucokinase to avoid this liability and to prevent hyperactivation of the enzyme," explains lead author David J. Lloyd. Liver-specific glucokinase activation is sufficient to promote antidiabetic effects and avoids the activation of pancreatic glucokinase, thereby avoiding hypoglycaemia.
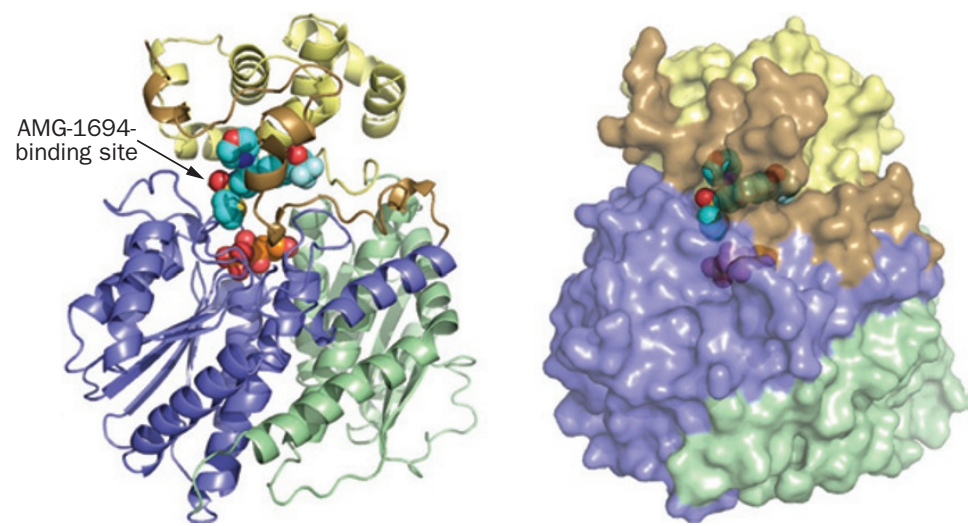

Co-crystal structure of human glucokinase regulatory protein with sorbitol-6-phosphate and AMG-1694. Permission obtained from Nature Publishing Group @ Lloyd, D. J. et al. Nature doi:10.1038/nature12724.

The main hurdle the investigators faced was that no small molecule had ever been identified that can disrupt the interaction between glucokinase and GCKR via direct targeting of the regulatory protein. Lloyd and colleagues screened for compounds that would disrupt this protein-protein interaction and now report the effects of two such small-molecule disruptors: AMG-1694 and AMG-3969. The compounds effectively lowered blood glucose levels in mouse models of T2DM (diet-induced obese, $o b / o b$ and $d b / d b$ mice) and in Zucker diabetic fatty rats.

One of the key aspects of the study was the resolution of the regulatory protein crystal structure of human GCKR and the identification of a distinct binding pocket within it, which was occupied by the small molecules. "Having that information enabled us to maximize occupancy of the pocket and improve features such as potency," says Lloyd, "and to show that a previously considered intractable target can be modulated by small molecules."

\section{Linda Koch}

\footnotetext{
Original article Lloyd, D. J. et al. Antidiabetic effects of glucokinase regulatory protein small-molecule disruptors. Nature doi:10.1038/nature12724
} 